\title{
A 4/3-approximation for TSP on cubic 3-edge-connected graphs
}

\author{
Nishita Aggarwal Naveen Garg Swati Gupta
}

September 21, 2018

\section{Introduction}

We consider the travelling salesman problem on metrics which can be viewed as the shortest path metric of an undirected graph with unit edge-lengths. Finding a TSP tour in such a metric is then equivalent to finding a connected Eulerian subgraph in the underlying graph. Since the length of the tour is the number of edges in this Eulerian subgraph our problem can equivalently be stated as follows: Given an undirected, unweighted graph $G=(V, E)$ find a connected Eulerian subgraph, $H=\left(V, E^{\prime}\right)$ with the fewest edges. Note that $H$ could be a multigraph.

In this paper we consider the special case of the problem when $G$ is 3-regular (also called cubic) and 3-edge-connected. Note that the smallest Eulerian subgraph contains at least $n=|V|$ edges. In fact, in the shortest path metric arising out of such a graph the Held-Karp bound for the length of the TSP tour would also be $n$. This is because we can obtain a fractional solution to the sub-tour elimination LP (which is equivalent to the Held-Karp bound) of value $n$ by assigning $2 / 3$ to every edge in $G$.

Improving the approximation ratio for metric-TSP beyond $3 / 2$ is a long standing open problem. For the metric completion of cubic 3-edge connected graphs Gamarnik et.al. [1] obtained an algorithm with an approximation guarantee slightly better than $3 / 2$. The main result of this paper is to improve this approximation guarantee to $4 / 3$ by giving a polynomial time algorithm to find a connected Eulerian subgraph with at most $4 \mathrm{n} / 3$ edges. This matches the conjectured integrality gap for the sub-tour elimination LP for the special case of these metrics.

\section{Preliminaries}

Let $n$ be the number of vertices of the given graph G. Let $d(x)$ denote the degree of x. A 2-factor in $G$ is a subset of edges $X$ such that every vertex has degree 2 in $X$. Let $\sigma(\mathrm{X})$ denote the minimum size of components of X. Given two distinct edges $e_{1}=x_{1} v$ and $e_{2}=x_{2} v$ incident on a vertex $v$, let $G_{v}{ }^{e_{1}, e_{2}}$ denote the graph obtained by replacing $e_{1}, e_{2}$ by the edge $x_{1} x_{2}$. The vertex $v$ is said to be split off. We call a cut $(S, \bar{S})$ essential when both $S$ and $\bar{S}$ contain at least one edge each.

We will need the following results for our discussion

Lemma 1 (Peterson[4]). Every bridgeless cubic graph has a 2-factor.

Lemma 2 (Mader[3]). Let $G=(V, E)$ be a $k$-edge-connected graph, $v \in V$ with $d(v) \geq k+2$. Then there exists edges $e_{1}, e_{2} \in E$ such that $G_{v}^{e_{1}, e_{2}}$ is homeomorphic to a k-edge-connected graph.

Lemma 3 (Jackson, Yoshimoto[2]). Let $G$ be a 3-edge-connected graph with $n$ vertices. Then $G$ has a spanning even subgraph in which each component has at least $\min (n, 5)$ vertices. 


\section{Algorithm}

Our algorithm can be broadly split into three parts. We first find a 2 -factor of the cubic graph that has no 3-cycles and 4-cycles. Next, we compress the 5-cycles into 'super-vertices' and split them using Lemma 2 to get a cubic 3-edge-connected graph $\mathrm{G}^{\prime}$ again. Repeatedly applying the first part on $G^{\prime}$ and compressing the five cycles gives a 2-factor with no 5-cycle on the vertices of the original graph. We 'expand' back the super-vertices to form $X$ that is a subgraph of $\mathrm{G}$. We finally argue that $X$ can be modified to get a connected spanning even multi-graph using at most 4/3(n) edges.

The starting point of our algorithm is Theorem 3 [2]. In fact [2] proves the following stronger theorem.

Theorem 1. Let $G$ be a 3-edge-connected graph with $n$ vertices, $u_{2}$ be a vertex of $G$ with $d\left(u_{2}\right)=3$, and $e_{1}=u_{1} u_{2} ; e_{2}=u_{2} u_{3}$ be edges of $G$. (it may be the case that $u_{1}=u_{3}$ ). Then $G$ has a spanning even subgraph $X$ with $\left\{e_{1}, e_{2}\right\} \subset E(X)$ and $\sigma(X) \geq \min (n, 5)$.

The proof of this theorem is non-constructive. We refer to the edges $e_{1}, e_{2}$ in the statement of the theorem as "required edges". We now discuss the changes required in the proof given in [2] to obtain a polynomial time algorithm which gives the subgraph $X$ with the properties as specified in Theorem 1. Note that we will be working with a 3-regular graph (as against an arbitrary graph of min degree 3 in [2]) and hence the even subgraph $X$ we obtain will be a 2-factor.

1. If $G$ contains a non-essential 3-edge cut then we proceed as in the proof of Claim 2 in [2]. This involves splitting $G$ into 2 graphs $G_{1}, G_{2}$ and suitably defining the required edges for these 2 instances so that the even subgraphs computed in these 2 graphs can be combined. This step is to be performed whenever the graph under consideration has an essential 3-edge cut.

2. Since $G$ is 3-regular we do not require the argument of Claim 6.

3. Since $G$ has no essential 3-edge cut and is 3-regular, a 3-cycle in $G$ implies that $G$ is $K_{4}$. In this case we can find a spanning even subgraph containing any 2 required edges.

4. The process of eliminating 4-cycles in the graph involves a sequence of graph transformations. The transformations are as specified in [2] but the order in which the 4-cycles are considered depends on the number of required edges in the cycle. We first consider all such cycles which do not have any required edges, then cycles with 2 required edges and finally cycles which have one required edge.

Since with each transformation the number of edges and vertices in the graph reduces we would eventually terminate with a graph, say $G^{\prime}$, with girth 5 . We find a 2 -factor in $G^{\prime}$, say $X^{\prime}$ and undo the transformations (as specified in [2]) in the reverse order in which they were done to obtain a 2-factor $X$ in the original graph $G$ which has the properties of Theorem 1 .

Suppose the 2-factor obtained $X$ contains a 5-cycle $C$. We compress the vertices of $C$ into a single vertex, say $v_{C}$, and remove self loops. $v_{C}$ has degree 5 and we call this vertex a super-vertex. We now use Lemma 2 to replace two edges $x_{1} v_{C}$ and $x_{2} v_{C}$ incident at $v_{C}$ with the edge $x_{1} x_{2}$ while preserving 3-edge connectivity. The edge $x_{1} x_{2}$ is called a super-edge. Since the graph obtained is cubic and 3-edge connected we can once again find a 2-factor, each of whose cycles has length at least 5. If there is a 5-cycle which does not contain any super-vertex or super-edge we compress it and repeat the above process. We continue doing this till we obtain a 2-factor, say $X$, each of whose cycles is either of length at least 6 or contains a super-vertex or a super-edge. 
In the 2-factor $X$ we replace every super-edge with the corresponding edges. For instance the superedge $x_{1} x_{2}$ would get replaced by edges $x_{1} v_{C}$ and $x_{2} v_{C}$ where $v_{C}$ is a super-vertex obtained by collapsing the vertices of a cycle $C$. After this process $X$ is no more a 2-factor but an even subgraph. However, the only vertices which have degree more than 2 are the super-vertices and they can have a maximum degree 4 . Let $X$ denote this even subgraph.

Consider some connected component $W$ of $X$. We will show how to expand the super-vertices in $W$ into 5-cycles to form an Eulerian subgraph with at most $\left\lfloor 4\left|W^{\prime}\right| / 3\right\rfloor-2$ edges, where $\left|W^{\prime}\right|$ is number of vertices in the expanded component. For each component we will use 2 more edges to connect this component to the other components to obtain a connected Eulerian subgraph with at most $\lfloor 4 n / 3\rfloor-2$ edges. Note that the subgraph we obtain may use an edge of the original graph at most twice.

We now consider two cases depending on whether $W$ contains a super-vertex.

1. $W$ has no super-vertices. Then, $W$ is a cycle with at least 6 vertices and hence Eulerian. Since $|W| / 3 \geq 2$ the claim follows.

2. $W$ has at least one super-vertex, say $s$. We will discuss the transformations for a single super-vertex and this will be repeated for the other super-vertices. Note that $s$ has degree 2 or 4 .

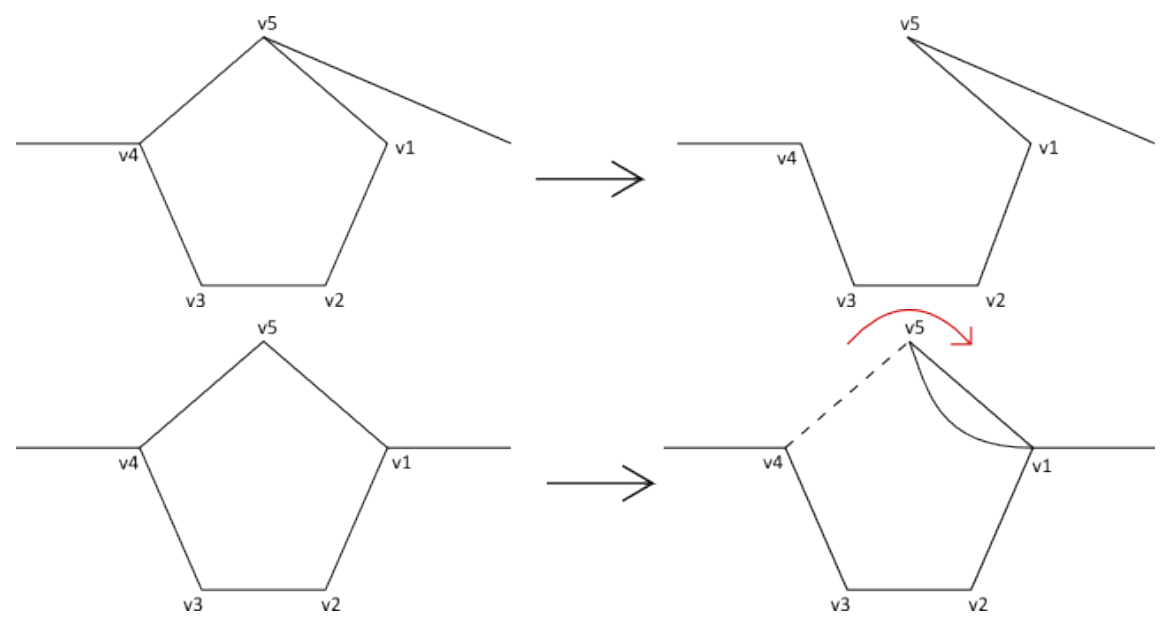

Figure 1: On Expanding a super-vertex with degree 2

If $s$ has degree 2, then the 2 edges incident on the 5-cycle corresponding to $s$ would be as in Figure 1 . In both cases we obtain an Eulerian subgraph. By this transformation we have added 4 vertices and at most 5 edges to the subgraph $W$.

Suppose the super-vertex $s$ has degree 4 in the component $W$. $W$ may not necessarily be a component of the subgraph $X$ as it might have been obtained after expanding a few super-vertices, but that will not effect our argument. Let $C$ be the 5-cycle corresponding to this super-vertex and let $v_{1}, v_{2}, v_{3}, v_{4}, v_{5}$ be the vertices on $C$ (in order). Further let $v_{i}^{\prime}$ be the vertex not in $C$ adjacent to $v_{i}$. Let $v_{5} v_{5}^{\prime}$ be the edge incident on $C$ that is not in the subgraph $W$.

We replace the vertex $s$ in $W$ with the cycle $C$ and let $W^{\prime}$ be the resulting subgraph. Note that by dropping edges $v_{1} v_{2}$ and $v_{3} v_{4}$ from $W^{\prime}$ we obtain an Eulerian subgraph which includes all vertices of $C$. However, this subgraph may not be connected as it could be the case that edges $v_{1} v_{2}$ and $v_{3} v_{4}$ 


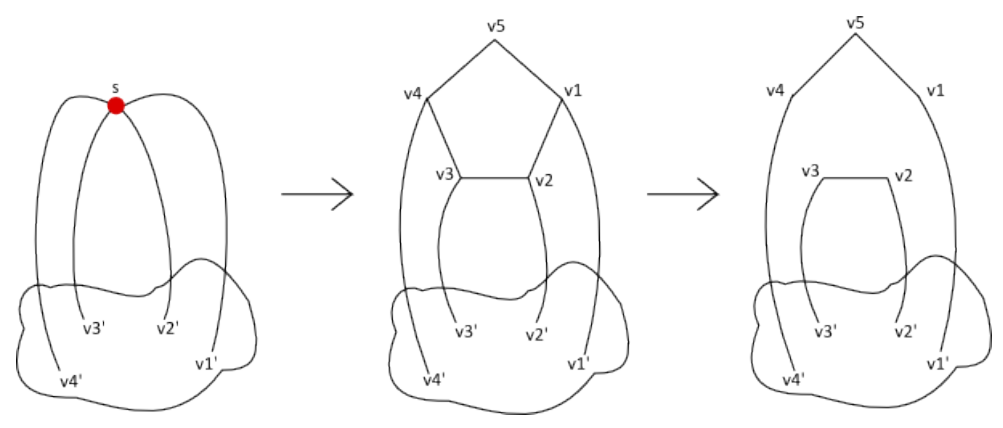

Figure 2: Expanding a super-vertex with degree 4 when $v_{1} v_{2}$ and $v_{3} v_{4}$ do not form a 2-edge-cut of the sub-graph constructed till now.

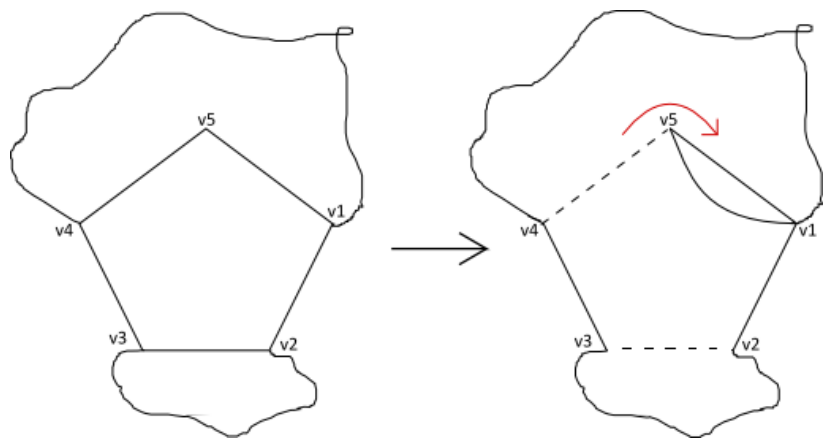

Figure 3: Expanding a super-vertex with degree 4 when $v_{1} v_{2}$ and $v_{3} v_{4}$ form a 2-edge-cut.

form an edge-cut in $W^{\prime}$. If this is the case then we apply the transformation as shown in Figure 3 . This ensures that $W^{\prime}$ remains connected and is Eulerian. Note that as a result of this step we have added 4 vertices and at most 4 edges to the subgraph $W$.

Let $W^{\prime}$ be the component obtained by expanding all the super-vertices in $W$. Suppose initially, component $W$ had $k_{1}$ super-vertices of degree $2, k_{2}$ super-vertices of degree 4 and $k_{3}$ vertices of degree 2 . This implies $W$ had $k_{1}+2 k_{2}+k_{3}$ edges. On expanding a super-vertex of degree 2 , we add 5 edges in the worst case. On expanding a super-vertex of degree 4 , we add 4 edges in the worst case. So, the total number of edges in $W^{\prime}$ is at most $6 k_{1}+6 k_{2}+k_{3}$ while the number of vertices in $W^{\prime}$ is exactly $5 k_{1}+5 k_{2}+k_{3}$. Note that $k_{1}+k_{2}+k_{3} \geq 5$ and if $k_{1}+k_{2}+k_{3}=5$ then $k_{1}+k_{2} \geq 1$. Hence, $2 k_{1}+2 k_{2}+k_{3} \geq 6$ and this implies that the number of edges in $W^{\prime}$ is at most $\left\lfloor 4\left|V\left(W^{\prime}\right)\right| / 3\right\rfloor-2$.

\section{Conclusions}

We show that any cubic 3-edge connected graph contains a connected Eulerian subgraph with at most $4 \mathrm{n} / 3$ edges. It is tempting to conjecture the same for non-cubic graphs especially since the result in [2] holds for all 3-edge connected graphs. The example of a $K_{3, n}$ demonstrates that this conjecture would be false. A $K_{3, n}$ is 3 -edge connected and any connected Eulerian subgraph contains at least $2 n$ edges. 


\section{References}

[1] David Gamarnik, Moshe Lewenstein, and Maxim Sviridenko. An improved upper bound for the tsp in cubic 3-edge-connected graphs. Oper. Res. Lett.

[2] Bill Jackson and Kiyoshi Yoshimoto. Spanning even subgraphs of 3-edge-connected graphs. Journal of Graph Theory, 62(1):37-47, 2009.

[3] W. Mader. A reduction method for edge-connectivity in graphs. Ann. Discrete Math, 3:145-164, 1978.

[4] J. Petersen. Die theorie der regularen graphen. Acta Math., 15:193-220, 1891. 